\title{
TERCERIZACIÓN Y REGULACIÓN LABORAL EN CHILE: UNA RECONSTRUCCIÓN DE LAS TRANSFORMACIONES DEL TRABAJO
}

\section{OUTSOURCING AND LABOUR REGULATION IN CHILE: A RECONSTRUCTION OF THE TRANSFORMATIONS OF WORK}

\author{
SEBASTIÁN PÉREZ SEPÚLVEDA ${ }^{1}$
}

\begin{abstract}
RESUMEN: El objetivo de este artículo es reconstruir el proceso de expansión y consolidación de la tercerización en Chile, en tanto operador analítico de las transformaciones del trabajo de las últimas décadas. El artículo pone en evidencia los resortes institucionales específicos que han sustentado este proceso, analizando la refundación neoliberal de la ley del trabajo operada en el contexto represivo de la dictadura (1973-1990), como también la ley 20.123 de 2006 que regula la tercerización y reproduce la lógica reformista desde 1990. Además de la reconstrucción analítica de este proceso, el artículo describe la evolución de las formas concretas de la tercerizaciónhasta la situación contemporánea.
\end{abstract}

Palabras clave: Tercerización; Subcontratación; Trabajo; Regulación; Chile.

ABSTRACT: The main aim of this article is to reconstruct the expansion and consolidation process of outsourcing in Chile, as an analytical operator of the transformations of work in recent decades. The article highlights the specific institutional support that has sustained this process, analyzing the neoliberal refounding of the labour law carried out in the repressive context of the dictatorship (1973-1990), and the law 20.123 of 2006 that regulates outsourcing and reproduces the reformist logic since 1990. In addition to the analytical reconstruction of this process, the article describes the evolution of concrete forms of outsourcing to their current status.

Keywords: Outsourcing; Subcontracting; Work; Regulation; Chile.

\footnotetext{
${ }^{1}$ Investigador postdoctoral, Institut de Recherche Interdisciplinaire en Sciences Sociales, IRISSO, Université Paris Dauphine-PSL. Master en ciencias sociales y Doctor en sociología, École des hautes études en sciences sociales, EHESS, París, Francia. Licenciado en sociología, Universidad de Chile. Correo electrónico : perez.sep.sebastian@gmail.comORCID: https://orcid.org/0000-00016096-7270.
} 


\section{INTRODUCCIÓN}

La tercerización transforma los mundos del trabajo. Ella multiplica la heterogeneidad de condiciones laborales no sólo a lo largo de cadenas globales de valor, sino también al interior de las unidades productivas, dando lugar a lo que David Weil denomina fissured workplace (WEIL, 2014). Sin embargo, la tercerización no es un concepto que describa una realidad definida, es más bien el nombre de un problema. La diversidad de prácticas que la tercerización encierra hacen de ella un fenómeno complejo que desafía las conceptualizaciones teóricas (BASUALDO; MORALES, 2014). En este sentido, una aproximación recurrente consiste en asociar la tercerización a la consolidación contemporánea del paradigma flexible y el movimiento de desconcentración productiva (ECHEVERRÍA, 2010). Sin embargo, la tercerización no es un fenómeno radicalmente nuevo. Algunas formas aparecen ya en el capitalismo industrial del siglo XIX (DIDRY, 2019), entre las que se encuentra el tâcheronnat en Francia o el sweating system en Inglaterra, formas de subcontratación en cascada, fundadas en el trabajo a destajo y que configuran un "sistema de explotación y opresión articulado jerárquicamente" (MARX, 1993, p. 620). La novedad reside más bien en que "la tercerización se ha transformado en un dispositivo privilegiado por los empresarios a la hora de reducir costos y lograr la descolectivización de la fuerza de trabajo, al mismo tiempo que se multiplican las formas en que aparece" (BATTISTINI, 2018, p. 318).

La centralidad de la tercerización en el capitalismo contemporáneo constituye por tanto no sólo un desafío teórico, sino también una prueba para las instituciones del trabajo. Si bien no existe una relación necesaria, se constata un vínculo empírico persistente entre tercerización, precarización e informalidad. La desestructuración de la relación salarial está frecuentemente a la base de una degradación de las condiciones del trabajo tercerizado, quedando muchas veces al margen de las regulaciones laborales y de la representación sindical. Aspectos que han adquirido mayor visibilidad con el auge de las economías de plataformas (ABDELNOUR; MÉDA, 2019).

Si el desafío es transversal, las formas concretas de tercerización y sus consecuencias específicas se declinan de acuerdo al carácter de las regulaciones laborales, tanto de aquéllas que definen la tercerización, como de aquéllas más generales que norman las relaciones entre capital y trabajo. En América Latina existen diferencias importantes en los modos de regulación de la tercerización y de representación sindical (BASUALDO; MORALES, 2014); diferencias que responden, de alguna manera, a la penetración desigual del neoliberalismo en la región.

En este sentido, el objetivo de este artículo es reconstruir analíticamente el proceso de expansión y consolidación de la tercerización en Chile durante las últimas décadas. Dos ideas están en el centro de este análisis. Por una parte, la tercerización ha contado con resortes institucionales específicos, los cuales se 
inscriben en un proceso más general de refundación neoliberal de la ley del trabajo, operada en el contexto represivo de la dictadura cívico-militar (1973-1990). Más tarde, la regulación de 2006 reproduce la lógica reformista desde 1990, buscando consolidar más que limitar esta práctica. Por otra parte, la tercerización constituye un operador analítico de las transformaciones del trabajo en el país, no tanto por su presencia estadística como por su alcance analítico, ya que las orientaciones principales que definen la regulación del trabajo se superponen de manera crítica en los contextos de tercerización ${ }^{2}$. Además de la introducción y la conclusión, el artículo se organiza en cuatro apartados. En el primero de ellos se exploran los resortes institucionales de la expansión y consolidación de la tercerización en Chile. Luego se sintetizan los rasgos principales de la subcontratación desde el Plan Laboral hasta la regulación de 2006 (ley 20.123). En el tercer apartado se analiza en detalle ésta última y sus principales consecuencias. Finalmente, en el cuarto apartado se describe el panorama actual de la tercerización en el país.

\section{RESORTES INSTITUCIONALES DE LA EXPANSIÓN DE LA TERCERIZACIÓN EN CHILE}

La tercerización no es nueva en la historia económica de Chile. Entre sus figuras tradicionales se encuentra el "enganchador", que alcanzó gran auge durante el período de explotación salitrera y cuya labor consistía en movilizar trabajadores del centro o del sur del país para ponerlos a disposición de las compañías mineras situadas en el norte, obteniendo una comisión por esta actividad. Aún es posible encontrar esa figura, principalmente en faenas agrícolas de temporada. Otras expresiones históricas de tercerización remiten al sector de la construcción como también al sector portuario, particularmente el trabajo temporal de estiba, además de la industria textil y de calzado, organizadas a través de pequeñas y medianas empresas (MONTERO, 1983). Sin embargo, todas estas figuras tradicionales ocupan un lugar más bien marginal en la matriz productiva nacional forjada durante el período desarrollista (1930-1973), dominada mayoritariamente por un modelo de empresa que integraba verticalmente en su interior el conjunto de las funciones productivas (MONTERO, 1983; DÍAZ, 1995). El escenario actual es diametralmente opuesto, toda vez que la tercerización está presente en todos los sectores productivos y en todas las regiones del país. La pregunta es cómo se desarrolló este cambio de escenario.

Lejos de responder a una adaptación progresiva de la organización de las empresas, tal como se ha observado en los países industrializados (CORIAT, 1990), el cambio de escenario en el caso chileno es más abrupto y radical. Éste se inscribe en el prematuro giro neoliberal operado desde 1975 a través de una serie de políticas de shock antiinflacionario y de transformaciones estructurales en el

\footnotetext{
${ }^{2}$ El análisis que sustenta este articulo forma parte de mi tesis doctoral sobre la consolidación de la subcontratación como operador analítico de las reconfiguraciones políticas del trabajo en Chile (PÉREZ SEPÚLVEDA, 2018)
} 
contexto de la dictadura cívico-militar $(1973-1990)^{3}$. En este marco, la apertura comercial y la eliminación de mecanismos de protección a la industria nacional, obligaron a las empresas a una racionalización productiva para hacer frente a la competencia internacional, haciendo de la tercerización un recurso central de adaptación. Sin embargo, existen dos modificaciones institucionales introducidas en este período que juegan un rol determinante a este respecto: la instauración de un impuesto al valor agregado y modificaciones trascendentales en la Ley del trabajo (ECHEVERRÍA, 2010).

En materia tributaria existían hasta 1973 dos tipos de impuestos indirectos: el impuesto sobre ventas y servicios y los impuestos aduaneros. Si estos últimos buscaban proteger la industria nacional, el primero incentivaba la integración vertical de funciones al interior de las empresas, toda vez que gravaban a una tasa variable cada una de las transacciones comerciales que participaban en las actividades productivas. Si los impuestos aduaneros comienzan a disminuir progresivamente luego del golpe de Estado, la reforma tributaria de 1975 introduce un cambio mayor: reemplaza el impuesto indirecto sobre ventas y servicios por el impuesto al valor agregado (IVA), instaurando una misma tasa y cuyo monto es inmediatamente deducido en las transacciones comerciales posteriores. Con ello, desparece el incentivo a la integración vertical y la externalización de funciones deviene un mecanismo de racionalización fundamental de las empresas para competir en una economía cada vez más abierta. A modo de ejemplo, un estudio del Instituto Chileno del Acero de la época sobre el fenómeno de la subcontratación en el sector de línea blanca señala que el principal incentivo para esta práctica es justamente la instauración del impuesto al valor agregado (INSTITUTO CHILENO DEL ACERO, 1978). Formando parte de una serie de políticas de shock destinadas a disminuir drásticamente la inflación y que entre otras medidas significó la reducción de 30\% del empleo público (GÁRATE, 2010), este cambio tributario constituye, en palabras de Magdalena Echeverría (2010), el "preludio" de la tercerización.

Sin embargo, el principal impulso hacia la tercerización está contenido en el llamado "Plan Laboral" de 1979, conjunto de decretos de ley (en adelante D.L.) que modifican aspectos estructurales de la legislación laboral chilena y que serán reagrupados más tarde en un nuevo Código del trabajo. El Plan Laboral

\footnotetext{
${ }^{3}$ Es importante señalar que si bien el viraje hacia el neoliberalismo en Chile es comparativamente prematuro, tampoco es inmediato al derrocamiento militar del gobierno de Salvador Allende. El neoliberalismo estaba presente entre los sectores reaccionarios que sustentaron el golpe de Estado, pero distaba de ser hegemónico. De hecho, el programa de transformaciones neoliberales - conocido popularmente como El Ladrillo y elaborado por los Chicago boys, conjunto de economistas chilenos formados en la Universidad Católica y posteriormente en la Universidad de Chicago a la sombra de Milton Friedman y Arnold Harberger - no se aplicará antes de 1975, momento en que se pone en práctica un "plan de recuperación económica" que se traduce en una política de shock para controlar la inflación. A partir de ese momento, los Chicago boys ocupan puestos clave en la administración del Estado, excluyendo definitivamente otras orientaciones políticoeconómicas, en particular el nacional-corporatismo representado por el comandante en jefe de la Fuerza Aérea Gustavo Leigh, quien será destituido en 1978 (GÁRATE, 2010; MONTERO,1997).
} 
corresponde a los D.L. 2.756 sobre organizaciones sindicales y 2.758 sobre negociación colectiva, pero los especialistas incluyen también modificaciones jurídicas que tratan sobre el derecho individual del trabajo: el D.L. 2.200 de 1978 sobre "Contrato de trabajo y la protección de los trabajadores", además del D.L. 2.950 de 1979 y la ley 18.081 de 1981, orientados a adecuar y armonizar el conjunto de la normativa laboral (UGARTE, 2014; ROJAS, 2007; NARBONA, 2015).

El Plan Laboral viene a llenar el vacío dejado por las medidas introducidas inmediatamente después del golpe de Estado, tales como el D.L. 133 del 29 de septiembre de 1973 que suspendió las normas relativas al ajuste de las remuneraciones, entre ellas la negociación colectiva y el derecho de huelga, además de disolver la Central Única de Trabajadores (CUT). Sin embargo, junto con llenar el vacío jurídico, el Plan Laboral introduce una refundación neoliberal de la norma del trabajo. Sin constituir una mera desregulación, lo que se impone es más bien una doble lógica: si bien se introducen mecanismos de flexibilidad al contrato de trabajo, éstos se acompañan de una sobrerregulación del derecho colectivo.

En este último aspecto, el D.L. 2.756 modificó la estructura sindical. La antigua segmentación entre sindicatos obreros, profesionales y agrícolas, es reemplazada por un esquemade cuatro sindicatos: de empresa, inter-empresa, de trabajadores independientes y de trabajadores de la construcción. Por otra parte, si en el antiguo modelo la afiliación sindical era obligatoria para los obreros, ésta deviene voluntaria. Sin embargo, el cuórum para formar sindicatos se eleva, al tiempo que libera la posibilidad de constituir más de un sindicato por empresa. Por su parte, el D.L. 2.758 impone un nuevo modelo de negociación colectiva. En la legislación anterior, la negociación ramal estaba reservada a los sindicatos profesionales. A partir de 1979, la negociación colectiva opera exclusivamente a nivel de empresa. Ella implica un empleador y el o los sindicatos de la empresa, además de "grupos de negociación" que los trabajadores puedan formar puntualmente para tal efecto. Dicho de otra manera, los sindicatos pierden la titularidad de la negociación colectiva. Además, el derecho de huelga queda contenido en el procedimiento de negociación colectiva, tiene una duración máxima de 60 días al término de los cuales los trabajadores son considerados en renuncia voluntaria y el empleador tiene la facultad de reemplazar los trabajadores en huelga. Tal como lo advierten Guillermo Campero y José Valenzuela (1984), la acción sindical es redimensionada: pierde su carácter de movimiento para devenir una acción profesional que interviene sólo en la determinación del precio del factor trabajo.

En paralelo, las modificaciones que afectan el derecho individualdel trabajo (D.L. 2.200 y la ley 18.081), introducen una flexibilidad a disposición unilateral del empleador. Por ejemplo, el empleador puede modificar unilateralmente los contratos en cuanto a la naturaleza de los servicios y el establecimiento en el cual éstos deban ejecutarse, junto con las jornadas de trabajo con un límite de 48 horas semanales y 12 horas diarias. Además, los contratos de trabajo llamados "especiales" (aprendizaje y trabajo a domicilio) quedan excluidos de la cobertura 
del salario mínimo, estableciéndose en su lugar el acuerdo "libre" entre las partes. Sin embargo, la modificación más profunda es la derogación de la ley 16.455 de 1966, llamada "ley de inamovilidad" que establecía un "sistema de estabilidad relativa de empleo" (ROJAS, 2007), al obligar al empleador a esgrimir una causa justificada de despido frente a un juzgado competente. En su lugar se reintroduce el despido libre (derogado por la ley 16.455), por medio de una carta de aviso de 30 días, dando lugar a una indemnización máxima de cinco salarios. Al mismo tiempo se introducen nuevas causas de despido que, de ser probadas, liberan al empleador del pago de toda indemnización. Es el caso de las causales "necesidades de la empresa" y aquellas incluidas en el D.L. 32 del 21 de septiembre de 1973 orientadas al disciplinamiento de la fuerza de trabajo en virtud - como señala el mismo decreto - de "la situación de emergencia que vive el país y la necesidad de reestablecer el principio y la práctica de la disciplina del trabajo en las actividades nacionales".

La refundación neoliberal de la norma del trabajo se completa con el D.L. 2.950, orientado, en palabras de José Piñera, Ministro del Trabajo de la época, a "sanear" el mercado laboral de una serie de "rigideces", entre ellas las disposiciones vigentes en materia de tercerización (PIÑERA, 1990). Hasta el golpe de Estado de 1973 existían tres disposiciones principales a este respecto. En primer lugar, una disposición que emana desde las llamadas Leyes sociales de 1924 y que establecía la responsabilidad subsidiaria del propietario de la faena respecto de las condiciones de trabajo de obreros subcontratados, exceptuándose el sector de la construcción y el caso en que el propietario fuese una persona natural. En segundo lugar, la ley 16.757 de 1968 prohibía la externalización de funciones consideradas permanentes de las empresas, como aquellas que forman parte de la actividad principal o la reparación de maquinaria. En tercer lugar, la ley 16.624 de 1967 que fijaba la igualdad de condiciones de trabajo, remuneraciones y derechos sociales entre trabajadores de planta y tercerizados del sector minero. Como señala Magdalena Echeverría, se trataba de disposiciones parciales, pero lo suficientemente eficientes para limitar la extensión de la tercerización (ECHEVERRÍA, 2010). No obstante, estas disposiciones (leyes 16.624 y 16.757) fueron derogadas por el D.L. 2.950 de 1979. En una visión retrospectiva, José Piñera describe el "saneamiento del mercado laboral" operado por este cuerpo legal:

El Decreto de Ley 2.950 que derogó el uso obligatorio de carnets profesionales en una infinidad de oficios y sectores de actividad, fue un gran aporte al saneamiento del mercado laboral del país. Ese mismo cuerpo legal derogó la Ley 16.757, que prohibía en forma absoluta que los trabajos inherentes a la producción principal y permanente de una industria fuesen efectuados por contratistas y concesionarios. Esta prohibición introducía a todas luces una rigidez inaceptable en la economía. (PIÑERA, 1990, p. 46) 
A partir de estos cambios institucionales, la tercerización queda jurídicamente liberada como práctica para adquirir una presencia creciente en la estructura productiva del país durante las décadas posteriores. Sin embargo, es importante insistir en dos elementos. En primer lugar, esta modificación hace parte de una transformación mayor que es posible identificar como una refundación neoliberal del trabajo, en la medida que modela el conjunto de la legislación laboral de acuerdo a las premisas de la economía monetarista y su visión estilizada del mercado, en tanto principio regulativo del orden social, no sólo económico 4 . Teoría económica que se proyecta como visión científica de lo real y que encuentra sus condiciones de verificaciones en las propias transformaciones que promueve (BOURDIEU, 2000), eliminando todo lo que considera como "rigideces" y desde donde se desprende una retórica naturalista ("sanear el mercado laboral"), no exenta de mesianismo ${ }^{5}$. Con todo, esta modelación de la legislación laboral no es menos autoritaria, toda vez que los dispositivos de flexibilidad quedan unilateralmente a la disposición de los empleadores frente a un sindicalismo jurídicamente desestructurado.

En segundo lugar, la refundación neoliberal del trabajo - al alero de la cual se destraba jurídicamente la tercerización laboral - precede y posibilita la transformación capitalista chilena que se desarrolla a partir de 1980. En efecto, lejos de toda naturalidad, esta transformación institucional anterior, junto con las políticas de privatización de empresas públicas y de los derechos sociales (salud, educación, previsión), genera las condiciones del viraje en la estrategia de desarrollo (de la industrialización a la exportación de materias primas) y de la reconversión productiva (MARTÍNEZ; DÍAZ, 1996). Varios análisis han mostrado que ésta última corresponde menos a un proceso de modernización de las empresas basada en la innovación tecnológica que a una adaptación fundada en la reducción de los costos laborales, en particular a través de estrategias de subcontratación y tercerización laboral (DÍAZ, 1995; AGACINO; RIVAS, 1995;ABRAMO; MONTERO; REINECKE, 1997).

\footnotetext{
${ }^{4}$ De acuerdo al análisis de Michel Foucault (2004), el neoliberalismo norteamericano - que se impone en Chile - a diferencia del neoliberalismo alemán, insiste menos en la generación de condiciones para el funcionamiento del mercado en el espacio económico que en la extensión de la racionalidad mercantil al conjunto de esferas sociales consideradas, en principio, no económicas. Es lo que ocurre en Chile con la privatización y mercantilización de los derechos sociales de educación, salud y previsión a partir de 1981.

${ }^{5} \mathrm{La}$ investigación de Cecilia Montero (1997) sobre la transformación empresarial chilena ha insistido en este punto. De acuerdo a su análisis, los economistas de Chicago se creían los portadores de la misión histórica de llevar al país al desarrollo, esta vez a partir de bases "científicas", en contraposición con lo que consideraban como "voluntarismo" y "artificialidad" de las políticas desarrollistas.
} 


\section{EXPANSIÓN Y PRECARIZACIÓN: TRES DÉCADAS DE TERCERIZACIÓN DESREGULADA (1979-2006)}

Si la tercerización constituye la pieza fundamental de la reconversión productiva de los años 1980, ésta se consolida durante los años 1990. Para una caracterización general de la subcontratación durante este período, una investigación ineludible es la que desarrollan Abramo, Montero y Reinecke al alero de la OIT (1997) ${ }^{6}$. Una primera constatación a este respecto es que la gran mayoría de las empresas emplean mecanismos de flexibilidad que precarizan el trabajo, como cadenas de subcontratación desiguales y verticales. Otros resultados apuntan al tipo de subcontratación y de empresa contratista.

En el primer caso se distingue entre subcontratación de bienes y/o servicios y subcontratación de fuerza de trabajo (que más tarde se llamará suministro de trabajo). Se señala que la subcontratación de servicios está presente en todos los sectores, mientras que la subcontratación de bienes es más recurrente en el sector agroexportador y forestal. La subcontratación de trabajo está presente también en estos últimos casos (donde operan "contratistas" y "enganchadores"), además del sector servicios y comercio. Por otra parte, se añade que, a la excepción de la producción textil, de calzado y de las industrias metalúrgica y metalmecánica, la externalización de funciones opera mayoritariamente al interior de las empresas principales. Más allá de las diferencias se constata que ya a mediados de la década del 90, la subcontratación es constitutiva de los principales sectores productivos del país.

Para caracterizar las empresas contratistas, la investigación retoma la clasificación de Álvaro Díaz (1995), considerando el grado de especialización y de autonomía respecto de las empresas principales. Se distinguen tres tipos. En primer lugar, las más numerosas son contratistas de capacidad primaria, que corresponden a trabajadores individuales o empresas de menor tamaño, dedicadas al ensamblaje de partes o al aprovisionamiento de servicio con escaso valor agregado. Su nivel de tecnología es limitado y el trabajo, aun cuando especializado, no es calificado. El caso típico corresponde al trabajo a domicilio feminizado de la industria textil o del calzado, pero se encuentra también en los sectores agroexportador, forestal, servicios y comercial. En segundo lugar, las contratistas especializadas y dependientes, que corresponden a pequeñas y medianas empresas cuyo grado de tecnología aplicada y de calificación de la fuerza de trabajo tienden a ser más elevados. Sin embargo, estas contratistas son dependientes de las empresas principales, en cuanto a asistencia técnica, créditos de capital de trabajo y especificaciones sobre el proceso productivo, los productos y los plazos a respetar. Son características del sector metalmecánico, en particular en la cadena de elaboración de electrodomésticos. En tercer lugar, las contratistas especializadas y autónomas, que se caracterizan por un nivel de calificación de la fuerza de trabajo más elevado y un

\footnotetext{
${ }^{6}$ Esta investigación reposa sobre estudios monográficos en los sectores agro-exportador, minero, forestal, servicios y comercial, además de la industria textil, de calzado y metalmecánica.
} 
grado mayor de autonomía respecto de las empresas mandantes. Estas contratistas son escazas y se concentran en el sector minero y el sector comercial.

Una década más tarde, la investigación de Claudio Ramos (2009) sobre la modernización de las empresas en Chile prolonga estos hallazgos. De las empresas estudiadas, 67,7\% subcontrata actividades realizadas anteriormente al interior de la empresa. De esa proporción, 86,6\% subcontrata "servicios secundarios" (mantención, seguridad, restauración, transporte), 50,5\% "servicios fundamentales" (administración de personal, contabilidad, servicios legales y de atención al cliente) y 57,1\% subcontrata actividades de la función principal. Otro dato relevante es que la proporción de fuerza de trabajo suministrada temporalmente varia entre $10 \%$ a $80 \%$ entre las empresas estudiadas. Además de estas constataciones, un aporte de esta investigación es la caracterización del tipo de relación que establecen las empresas. Se estima que las empresas principales están en relación con un promedio de 3,6 empresas contratistas, relación que puede tomar tres formas. En primer lugar, relaciones esporádicas, que engloban un tercio de la muestra y son más próximas de las relaciones de mercado, toda vez que los vínculos obedecen a transacciones comerciales. En segundo lugar, relaciones percibidas como estables, que corresponden al $45 \%$ de los casos, donde no se observa transferencia de información y conocimiento, la atención se concentra en la evaluación del producto antes que en los procesos internos de las contratistas y el mecanismo de resolución de problemas es el reemplazo de éstas últimas. Finalmente, las redes de empresas, que abarcan $25 \%$ de los casos, donde el elemento característico es el vínculo de propiedad entre las empresas, el cual es "condición del refuerzo de la relación en materia de información, de coordinación y de apoyo técnico" (RAMOS, 2009, p. 164). El caso típico corresponde a empresas pertenecientes a un mismo holding.

De este panorama general es posible destacar tres elementos. En primer lugar, la extensión de la subcontratación como un rasgo central de la estructura productiva del país. En segundo lugar, esta generalización está lejos de la especialización flexible, toda vez que las empresas externalizan en proporción considerable actividades principales y las contratistas son mayoritariamente empresas de capacidades primarias con un uso intensivo de fuerza de trabajo especializada, pero poco calificada. En tercer lugar, esta generalización está también lejos de una lógica de redes horizontales, toda vez que se constata que las relaciones entre empresas son, por una parte, frágiles, próximas de relaciones de mercado $\mathrm{y}$, por otra, ampliamente verticales y desiguales en términos de transferencia de tecnología y de autonomía. La trayectoria chilena evidencia así la consolidación de "redes piramidales" (WORMALD, 1999), en las que la subcontratación apunta fundamentalmente a la reducción de costos laborales, lo que se traduce en precarización del trabajo tercerizado.

A este respecto, las investigaciones de los años 90 enfatizaron la opacidad del fenómeno, lo que se tradujo en escasez de datos y fuentes de información 
sistematizada de carácter general para precisar el alcance y las consecuencias sociales de la tercerización. En este sentido, la profundidad alcanzada por los estudios sectoriales y monográficos ${ }^{7}$ no es replicada por las encuestas nacionales de fines de la década del 90, las cuales si bien clarifican la magnitud de la externalización, permanecen a un nivel superficial del fenómeno. En cualquier caso, en materia de consecuencias sociales de la tercerización durante este período resultan clave las investigaciones de Magdalena Echeverría (1997, 2010), quien identifica cinco factores que determinan la precariedad de la fuerza de trabajo tercerizada: el objeto de externalización (producción o fuerza de trabajo), el lugar de operación de la externalización (dentro o fuera de la empresa principal), el grado de especialización técnica de la contratista, la duración de la tercerización y el grado de coordinación del conjunto de actividades internas y externas, lo que permite determinar las responsabilidades patronales. Dado que las prácticas concretas de tercerización son complejas, Magdalena Echeverría elabora dos tipos ideales. En un extremo, un modo de tercerización donde las probabilidades de precarización son más bien reducidas, el cual supone una subcontratación de producción (bienes o servicios), orientada a actividades complementarias, pero con un grado elevado de especialización técnica, de duración determinada y que se lleva a cabo al exterior de la empresa principal. Al otro extremo, un tipo de tercerización más precaria corresponde al suministro de trabajo poco especializado y calificado, destinado a la realización de actividades permanentes que forman parte de la línea productiva principal de la empresa principal. En este caso, las responsabilidades patronales tienden a ser más difusas y se condicen con remuneraciones más bajas del personal tercerizado, sumadas al pago irregular de las cotizaciones sociales. Por supuesto, la precariedad laboral no se encuentra solamente en este último caso, ella transita en diversos grados entre las múltiples formas de tercerización. Recordemos, además, que las contratistas con alto grado de especialización y autonomía son más bien escazas en la estructura productiva chilena.

Otras investigaciones asocian la precariedad al lugar ocupado en las cadenas de subcontratación, en cuya periferia intervienen microempresas y trabajo a domicilio, donde la condición salarial es más bien frágil, incluso inexistente, lo que se traduce en precariedad extrema del empleo e intensificación del trabajo (AGACINO, 2007).

En definitiva tal como señala el jurista Diego López, especialista en la materia:

En la práctica, la mayoría de las veces los trabajadores subcontratados son una fuerza de trabajo secundaria, peor remunerada, disponible para efectuar un reemplazo, colaborar ante un incremento de demanda o para ser derechamente incorporada en forma permanente al proceso productivo,

\footnotetext{
${ }^{7}$ Sin ánimo de exhaustividad, es importante destacar las investigaciones realizadas por el Programa Economía del Trabajo (PET) en varios sectores, entre ellos, en el sector forestal (ESCOBAR; LÓPEZ, 1996) y en el sector minero (AGACINO; GONZÀLEZ; ROJAS, 1998).
} 
sustituyendo la contratación directa de trabajadores por un costo menor. Es normal que trabajadores subcontratados que realizan las mismas funciones, en los mismos puestos de trabajo y en las mismas jornadas laborales que los trabajadores directamente contratados, ganen, sin embargo, remuneraciones significativamente menores; incluso muchas veces recibiendo sólo el salario mínimo. (LÓPEZ, 2008, p. 18)

A estas condiciones precarias se agregan las consecuencias de la segmentación de los colectivos de trabajo, como resultado de la externalización productiva. A este respecto, el efecto de la norma del trabajo en Chile es particularmente sensible, dado que la delimitación de las negociaciones colectivas a nivel de las empresas deviene un mecanismo institucional que refuerza la fragmentación de los trabajadores tercerizados, obligándolos a negociar separadamente con cada empresa contratista, pese a pertenecer a una misma cadena de subcontratación y ser, frecuentemente, dependiente de la misma empresa mandante (AGACINO, 2007; ECHEVERRIA, 2010; LÓPEZ, 2008).

En suma, utilizando las distinciones de Sophie Béroud y Paul Bouffartigue (2009), es posible plantear que la tercerización en Chile produce simultáneamente una precarización del empleo, vinculada a la inestabilidad de los contratos de trabajo flexible y menores condiciones salariales, una precarización del trabajo, en la medida que a las condiciones de seguridad ysalud laboral a menudo mínimas, se agregan niveles de especialización y de calificación limitadas que se traducen en altos grados de intensificación del trabajo $\mathrm{y}$, finalmente, una precarización de las capacidades de organización y de representación colectiva de los trabajadores implicados en estos regímenes de trabajo, donde las limitaciones jurídicas de la ley laboral refuerza una fragmentación de hecho. Tal es el panorama de la tercerización hacia 2006, momento en que se introduce una nueva regulación jurídica.

\section{LA INSTITUCIONALIZACIÓN DE LA TERCERIZACIÓN: PRINCIPIOS, SOMBRAS Y TENSIONES}

Antes de analizar la ley 20.123 que regula la tercerización en Chile, es necesario evocar algunos elementos de contexto que permitan comprender el carácter de esta regulación. En ese sentido, la transición política de 1990 no trajo consigo una transformación de las estructuras institucionales heredadas de la dictadura, entre ellas en materia laboral. En ello influye la Constitución política de 1980 que configura un régimen político con limitada expresión popular y mayorías calificadas para reformas de leyes de rango constitucional que regulan varios aspectos clave de la organización socio-económica del país (GARRETÓN, 2004). En ese marco se desarrolla una política de consensos orientada a asegurar la gobernabilidad del régimen, lo que se tradujo, en el caso de la Concertación (coalición de centro-izquierda en el poder entre 1990 y 2010), en un reformismo 
limitado respecto de la institucionalidad heredada, acompañada de una política social focalizada y subsidiaria, orientada a la reducción de los niveles de pobreza (FFRENCH-DAVIS; STALLINGS, 2001). Así, por más que el trabajo haya sido considerado central en el sostenimiento del régimen democrático, tal como lo aseguraba el programa original de la Concertación, las reformas introducidas fueron bastante parciales respecto del Plan Laboral de 1979 (UGARTE, 2014).

Sin embargo, el reformismo limitado no se explica solamente por el autoritarismo del régimen político. Allí interviene también la correlación de fuerzas sociales en la coyuntura transicional. Si bien el movimiento sindical jugo un papel relevante en la lucha contra la dictadura, se trató de un rol más bien simbólico al contribuir a un movimiento social mucho más diverso. Lo que no es casual, ya que la refundación neoliberal de la ley del trabajo y el carácter de la transformación económica (externalización productiva, desindustrialización, disminución y precarización del empleo público), habían minado las bases históricas de estructuración de los actores laborales. Por esta razón, si bien los niveles de sindicalización alcanzaban en 1990 cerca del 20\%, se trataba de un sindicalismo orgánicamente desestructurado (DRAKE, 2003) ${ }^{8}$. Por el contrario, las políticas de privatización de empresas y servicios públicos - que se prolongaron durante la transición política - dieron lugar a influyentes grupos económicos, con presencia y control monopólico en los principales sectores de la economía nacional. De esta manera, a diferencia del sindicalismo, el empresariado enfrentó el proceso de transición en situación de hegemonía, considerado el héroe de la modernización económica del país (MONTERO, 1997).

Luego de las experiencias de concertación social a inicios de la transición entre el gobierno, la CUT, la CPC (Confederación de la Producción y del Comercio) y los avances parciales de la primera reforma laboral, el proyecto de reforma presentado en el segundo gobierno de la Concertación - que modificaba aspectos nodales del Plan Laboral al extender la negociación colectiva a un nivel supra-empresa (ramal o territorial), además de prohibir el reemplazo de trabajadores en huelga - fue rechazado en el Parlamento. En ausencia de consensos políticos en materia laboral, la reforma laboral de 2001 (Ley 19.759) fue más acotada y avanzó sobre otros aspectos, como la regulación de nuevas formas de flexibilidad, considerando el modelo de relaciones laborales ya instalado (CAMPERO, 2007). En consecuencia, de lo que se tratará en adelante es de adecuar los derechos laborales a la competitividad de las empresas. La problemática laboral, otrora discursivamente central en la consolidación democrática, adquiere a partir de ese momento un carácter sectorial.

\footnotetext{
${ }^{8}$ Sobre esto, no hay que perder de vista que la Central Unitaria de Trabajadores, fundada en 1988, estuvo bajo la hegemonía de los principales partidos de la Concertación (la Democracia Cristiana y el Partido Socialista), hasta 2012 cuando asumió Bárbara Figueroa, perteneciente al Colegio de Profesores y militante del Partido Comunista.
} 
Lo anterior se manifiesta en las principales modificaciones introducidas a partir de 1990. A nivel individual es destacable la institucionalización del salario mínimo en diversos sectores de actividad desde el inicio de la transición, además del aumento del margen de indemnización por despido de cinco a once salarios. Sin embargo, se mantiene la causal de despido por "necesidades de la empresa". Desde 2001, la jornada laboral disminuye de 48 a 45 horas semanales y se introduce un sistema de seguro de desempleo que opera a partir de cuentas individuales (de un modo similar al sistema de capitalización individual a nivel previsional instaurado en 1981). En paralelo, se institucionalizan otros mecanismos de flexibilidad, apuntando discursivamente a fomentar el empleo femenino y juvenil: es el caso de la jornada parcial, la polifuncionalidad y el teletrabajo. En cuanto al derecho colectivo, desde 1990 disminuye del cuórum para formar sindicatos y se institucionaliza del funcionamiento de centrales sindicales. A partir de 2001, las negociaciones colectivas inter-empresa dejan de estar prohibidas, pero deben contar con el acuerdo voluntario de los empleadores implicados, lo que las vuelve, en la práctica, marginales, si no inexistentes. Finalmente, en materia de derecho de huelga, se elimina su duración máxima y, más tarde, se condiciona la facultad de reemplazo de los trabajadores movilizados al pago de un bono por parte del empleador (ROJAS, 2007; NARBONA, 2015).

En virtud de estos elementos es evidente que la práctica reformista consolidó el Plan Laboral de 1979: se institucionalizan nuevos mecanismos de flexibilidady se mantienen aspectos esenciales del modelo de relaciones laborales. Si bien el sentido explícito de las reformas ha sido resguardar los derechos de los trabajadores frente a lo que se asume como la necesaria competitividad de las empresas, so pena de afectar el crecimiento y el nivel de empleo, la protección jurídica se ha restringido a un plano individual. El resultado ha sido la institucionalización de dispositivos ya empleados por las empresas, en un afán de formalizar las relaciones salariales y adecuar los contratos y las responsabilidades patronales a la normativa vigente. Sin embargo, al mantener un sindicalismo de empresa con una limitada cobertura de negociaciones colectivas, el conflicto laboral ha tendido a la judicialización (UGARTE, 2014).

En este marco general tiene lugar la regulación de la tercerización en 2006. Sin embargo, este proceso no obedece a la iniciativadel propio sistema político, obedece más bien a una coyuntura específica, marcada por la irrupción de una movilización y huelga de trabajadores subcontratados de la minera estatal Codelco, entre diciembre 2005 y enero 2006, en pleno proceso de elección presidencial. Las demandas de los trabajadores tercerizados apuntaban a una mejora de las condiciones laborales y un bono en retribución de las ganancias de la minera, asociadas al precio histórico que había alcanzado el cobre durante la primera mitad de la década. A partir del compromiso adquirido para con la movilización durante la campaña de segunda vuelta presidencial, el gobierno de Michelle Bachelet le entrega urgencia al proyecto de ley en materia de subcontratación que había sido 
ingresado en 2002 al Parlamento. Luego de meses de discusión y de la intervención del Tribunal Constitucional - volveremos sobre ello - finalmente se promulga la ley 20.123 que regula la tercerización el 5 de octubre de 2006.

La ley 20.123, conocida como "ley de subcontratación", regula en realidad dos regímenes de trabajo tercerizado: el trabajo en subcontratación y el suministro temporal de trabajadores. Una diferencia importante entre estas dos formas de tercerización reside en la composición de las relaciones de subordinación y dependencia. En el caso de la subcontratación, éstas se mantienen en la figura de un solo empleador: la empresa contratista. Por el contrario, estas dimensiones se separan en el caso del suministro temporal, ya que los trabajadores suministrados son dependientes de la empresa intermediaria, denominada Empresa de servicios transitorios, pero están subordinados al control de la empresa usuaria. Se trata, por tanto, de una relación triangular explícitamente definida y legitimada.

Además de la definición de ambos regímenes de trabajo, la ley 20.123 modifica las responsabilidades patronales. En el caso de la subcontratación, la empresa principal es, en principio, solidariamente responsable de las condiciones salariales y previsionales de los trabajadores tercerizados. Sin embargo, la responsabilidad subsidiaria no desaparece, ya que la empresa principal puede acogerse a ella si cumple con informarse regularmente del pago de los salarios y las cotizaciones previsionales por parte de las empresas contratistas. El principio es asociar el grado de responsabilidad de la empresa mandante al grado de control e implicación de ésta última en la operación de las empresas contratistas. Sin embargo, la empresa principal es responsable de las condiciones de seguridad y de salud laboral de los trabajadores de planta y tercerizados interviniendo en las faenas de trabajo bajo su control o propiedad. Por el contrario, en el caso del suministro temporal de trabajadores, la empresa principal o usuaria es siempre subsidiariamente responsable de las obligaciones salariales y previsionales de la fuerza de trabajo suministrada. En este caso, la responsabilidad principal recae en la Empresa de servicios transitorios, la cual debe operar exclusivamente bajo este giro comercial, ser autónoma respecto de la empresa usuaria y estar inscrita enun registro público de la Dirección del Trabajo.

Si la subcontratación no encuentra mayores limitaciones a su ejercicio, el suministro de trabajadores, en cambio, debe respetar su carácter temporal, respondiendo a circunstancias específicas de las empresas usuarias y sin sobrepasar 180 días de ejercicio. El no cumplimiento de las condiciones determinadas por la ley 20.123 implica la consideración del personal tercerizado como parte del personal de planta, obligando a su contratación por parte de la empresa principal o usuaria.

Como resulta evidente, la ley 20.123 no reintroduce las disposiciones derogadas por el D.L. 2.950 de 1979. Es más, lejos de limitar el ejercicio de la tercerización, el espíritu de la norma ha sido más bien promoverla bajo un marco jurídico determinado. Se reproduce, de esta manera, la misma lógica reformista de 
institucionalización sistemática de nuevas formas de flexibilidad, legitimando, en este caso, una práctica hasta ese momento ilegal, como el suministro temporal de trabajadores.

Con todo, es posible identificar cuatro elementos problemáticos de la ley 20.123 que serán fuente de tensiones importantes a partir de 2007. En primer lugar, la ausencia de disposiciones que permitan asegurar la igualdad de condiciones entre trabajadores de planta y tercerizados, en circunstancias que la tercerización ha operado como un mecanismo de reducción de costos laborales. De esta manera, pese a que el discurso de promulgación de la "ley de subcontratación" defendía el haber acabado con los "trabajadores de segunda categoría", lo cierto es que se institucionaliza la desigualdad estructural de los regímenes de trabajo tercerizado (ECHEVERRÍA, 2010). Otro aspecto problemático de la normativa dice relación con la ausencia de limitaciones respecto de las funciones que pueden ser externalizadas, pudiendo incluir, como es un caso recurrente, las funciones que forman parte de la actividad principal de las empresas. Además de cuestionar el carácter especializado de esta externalización, la tercerización de las actividades principales revela un sentido de reducción de costos laborales que se traduce en la precarización de las condiciones laborales del personal tercerizado (BASUALDO; MORALES, 2014). Junto con ello, plantea la interrogante respecto de lo que define efectivamente una empresa, lo que en Chile no tiene sólo un carácter teórico y constituye un tercer elemento problemático de la ley 20.123.

A diferencia del derecho comercial que no reconoce el estatus jurídico de la empresa (ARTEAGA, 2002), el Código laboral define la empresa a partir de una identidad legal determinada, lo que corresponde a un RUT: Rol Único Tributario (UGARTE, 2013) $^{9}$. El proceso legislativo de la ley 20.123 incluía una indicación que proponía la eliminación de la "identidad legal determinada" en la definición de empresa para efectos de esta norma, buscando limitar la práctica de subdivisión formal de las empresas en diversos RUT; práctica conocida popularmente como multirut, considerada abusiva por las consecuencias individuales y colectivas que produce. Por una parte, esta subdivisión permite la transferencia de la gestión de la fuerza de trabajo desde las empresas matrices a otras específicamente creadas para este efecto. Así, el pago de gratificaciones y otras obligaciones salariales son reconducidas a uno o varios terceros - empresas-rut - disminuyendo significativamente su magnitud. Por otra parte, la subdivisión de empresas se traduce inmediatamente en fragmentación sindical. Dado que la negociación colectiva está en la práctica limitada a nivel de empresa, la fuerza de trabajo así tercerizada debe negociar con su empleador o empresa-rut, blindando la identidad legal determinada que acumula las ganancias (AGACINO, 2007).

\footnotetext{
${ }^{9}$ El artículo 3 del Código del trabajo sostiene: "Para todos los efectos de la legislación laboral y de la seguridad social, se entiende por empresa toda organización de medios personales, materiales e inmateriales ordenados bajo la dirección de un empleador, para fines económicos, sociales, culturales o benéficos, dotada de una identidad legal determinada".
} 
Sin embargo, luego de aprobada la ley 20.123, senadores de la derecha presentaron un requerimiento al Tribunal Constitucional en contra de la indicación que eliminaba la identidad legal determinada. El fallo del Tribunal estima la inconstitucionalidad de esta modificación, pero en lugar de atender a los argumentos de este requerimiento (limitar la libertad de empresa), basa su decisión en un "vicio de procedimiento": dado que la definición de empresa compromete el pago de cotizaciones previsionales de los trabajadores, se trató para el Tribunal de una disposición que afectaba las normas de seguridad social, cuya modificación es de iniciativa exclusiva del Presidente de la República, en circunstancias que la indicación fue presentada por miembros de la Comisión de trabajo de la Cámara de diputados en enero de $2006^{10}$. De esta manera, la ley 20.123 fue promulgada manteniendo la definición legal de empresa, dejando abierta la problemática ${ }^{11}$.

Finalmente, un último elemento problemático concierne las atribuciones de la Dirección del Trabajo. De acuerdo a la ley 20.123, esta institución es la encargada de fiscalizar el cumplimiento de la normativa, lo que implica la evaluación de la realidad del trabajo tercerizado y, con ello, la interpretación de la validez de los contratos de trabajo. Sin embargo, esta interpretación sería una atribución exclusiva de los Tribunales del trabajo. A partir de 2007, la Dirección del Trabajo organiza un plan de fiscalización, comenzando por las empresas de retail y entre las irregularidades más recurrentes se encuentra que los trabajadores subcontratados operan, en la práctica, bajo la subordinación de las empresas principales, lo que supone su inclusión al personal de planta. Las empresas presentan requerimientos a los Tribunales, aludiendo la extensión de facultades de la Dirección del Trabajo, lo que es acogido. Los Tribunales fallan sobre las competencias de la Dirección del Trabajo, pero no se pronuncian sobre las irregularidades detectadas, por lo que queda abierta la posibilidad para que los trabajadores afectados puedan proseguir judicialmente estos procesos, pero a título individual (LÓPEZ, 2008). Una vez más, los conflictos laborales tienden así a la judicialización.

El conjunto de estos aspectos definen un terreno problemático que será fuente de tensiones importantes entre 2007 y 2008, donde intervienen distintos acontecimientos que traen el trabajo al centro debate público y la tercerización operará como catalizador relevante. Por razones de espacio sólo evocaremos cada uno de estos acontecimientos para relevar su importancia. En primer lugar, entre

\footnotetext{
${ }^{10}$ Cada una de las etapas legislativas de la ley 20.123 están consignadas en el documento "Historia de la Ley 20.123", elaborado por la Biblioteca del Congreso Nacional, disponible en: www.bcn.cl

${ }^{11}$ Más tarde, la ley 20.760 de 2014 buscó resolver el problema del multirut. La jurisprudencia en materia laboral había fallado supeditando la identidad legal a la doctrina del levantamiento del velo corporativo o el principio de primacía de la realidad (Ugarte, 2013), sin embargo, lo que hace la ley 20.760 es más bien reforzar la figura del empleador, estableciendo una serie de elementos definitorios más allá de la identidad legal, tales como una dirección común, la similitud o complementariedad de productos o servicios y la figura de un controlador común. Si bien estas disposiciones permiten la imputación de responsabilidades más allá de la empresa-rut que figura en el contrato de trabajo, en la práctica implica más dificultades a los trabajadores al momento de probar, frente al Tribunal del trabajo, que dos o más RUT son en realidad una empresa.
} 
2007 y 2008 tiene lugar una serie de movilizaciones sindicales y huelgas en la economía exportadora (industria minera, forestal, salmón y agroindustria), que tienen a trabajadores y trabajadoras tercerizados como protagonistas (LÓPEZ, 2008; ARAVENA; NÚÑEZ, 2009). Las demandas apuntan al respeto de la ley 20.123 y la mejora de condiciones laborales respecto del personal de planta. Entre los aspectos destacados de estas movilizaciones es posible mencionar, por un lado, la agrupación autónoma en federaciones y confederaciones sindicales propias de trabajadores tercerizados y, por otro, la renovación de los repertorios de acción (huelga, acción directa, movilización en los centros urbanos), encaminados a imponer negociaciones colectivas directamente con las empresas mandantes, desplazando de esta manera los límites de la ley laboral. Con resultados dispares, algunas de estas experiencias logran establecer las negociaciones colectivas con las empresas principales (Codelco, Holding Arauco), alcanzando gran presencia mediática y constituyéndose en las movilizaciones obreras más importantes desde 1990.

En segundo lugar, a partir del rol de mediación ejercido en estas movilizaciones, la Iglesia Católica propone la idea de un "salario ético", de un monto mucho más elevado que el salario mínimo de la época, lo que genera una amplio debate público en torno a esta materia, en la medida que vincula la crítica a las desigualdades sociales con una discusión en torno al valor del trabajo, lo que se cristaliza, de alguna manera, en la tercerización como paradigma de las desigualdades estructurales del mundo del trabajo.

En tercer lugar, la Dirección del Trabajo había continuado su labor fiscalizadora en el sector minero, particularmente en Codelco, dictaminando la inclusión 4800 trabajadores tercerizados al personal de planta. Al igual que las empresas fiscalizadas anteriormente, Codelco impugna lo que considera una extensión de facultades de la Dirección del Trabajo a nivel judicial, generando una tensión política interna al propio gobierno, en la medida que los Ministros de Hacienda y de Minería forman parte del directorio de Codelco. Pese al intento de mediación de la Presidencia, el resultado judicial fue favorable a la minera estatal, afectando la legitimidad de la nueva regulación. En ese mismo contexto, nuevas movilizaciones de obreros tercerizados de Codelco se desarrollan, no sólo en señal de protesta frente a la controversia con la Dirección del Trabajo, sino también en contra de la empresa estatal por no respetar el "Acuerdo Marco" de 2007 que traducía mejoras laborales. Para evitar una nueva negociación colectiva supra-empresa el propio gobierno, a través de los Ministros del Trabajo y de Interior, negocia directamente con la representación sindical tercerizada (LÓPEZ, 2008).

Estos tres acontecimientos, sin duda vinculados entre sí, generan un desborde plural de la representación dominante del trabajo que se había consolidado a partir de 1990. No sólo reaparece la huelga obrera, sino que ésta es encarnada por nuevos sujetos laborales, emergidos desde las propias transformaciones del trabajo. Asimismo, se desplazan los límites de las negociaciones colectivas, sobrepasando 
el nivel de empresa e involucrando la intervención del gobierno, rompiendo así el divorcio entre relaciones laborales y política que había instaurado el Plan Laboral. De la misma manera, la problemática del trabajo vuelve al debate público, desafiando su comprensión sectorial y desafiando también su reducción al empleo, portando nuevas problemáticas como las desigualdades estructurales del trabajo tercerizado.

Sin embargo, este desborde es transitorio. Para enfrentar esta problemática creciente del trabajo, el gobierno instala un Consejo Asesor, compuesto de representantes de distintos actores políticos, académicos y de la sociedad civil, encargado de elaborar propuestas de política pública ${ }^{12}$. Si bien el Consejo no alcanza consenso en todas las materias - actualizando de alguna manera las tensiones de fines de los años 90 - produce, a nuestro juicio, un efecto de normalización (en un sentido foucaultiano) del debate público, recentrando la problemática del trabajo en términos de empleo. Patricio Meller, economista y presidente del Consejo así lo explica en una entrevista publicada en La Nación el 10 de mayo de 2008:

A la demanda de un salario ético, nosotros respondemos con la subvención al ingreso del trabajo [...] Lo que se propone es más eficiente, menos caro y mucho menos perturbador para el mercado del trabajo. Lo que está en juego es la idea que la mejor política social es que la gente trabaje [...] El tema de la equidad no pasa solamente por la negociación colectiva [...] Si planteamos que el fisco entregue los recursos es porque creemos que el objetivo central es crear empleo. Los empresarios son aquellos que ofrecen este empleo.

Como es evidente, la problemática se vuelve a reducir a la combinación de empleo (mercado del trabajo) y Estado subsidiario, es decir, los márgenes hegemónicos del modelo de sociedad heredado desde la dictadura. Si bien la discusión pública en torno al trabajo tiende a cancelarse a partir de ese momento, el final del primer gobierno de Michelle Bachelet (2006 - 2010) marca el agotamiento del proyecto político de la Concertación (GARRETÓN, 2012). Lo que adviene es un proceso de recomposición política, con la vuelta de la derecha al poder, la emergencia de nuevos referentes políticos y la recuperación de la acción colectiva, con expresiones relevantes en el movimiento estudiantil, movimientos ecologistas, feministas, contra el sistema de pensiones y también cierta recuperación de los niveles de sindicalización y de la actividad huelguista (OBSERVATORIO DE HUELGAS LABORALES, 2017).

\footnotetext{
${ }^{12}$ No era la primera vez, el gobierno de Michelle Bachelet había ya puesto en práctica varios Consejos asesores para abordar diversas problemáticas (GARRETÓN; CRUZ; AGUIRRE, 2012)
} 
En este contexto, el último gobierno de Michelle Bachelet (2014 - 2018), que pretendía de alguna manera encarnar procesos de transformación estructural, presenta en 2016 una nueva reforma laboral que, apuntando discursivamente a "emparejar la cancha", proponía recuperar la titularidad sindical, además de eliminar definitivamente el reemplazo de trabajadores en huelga. Los resultados son, no obstante, limitados. Si bien, la facultad de reemplazar los trabajadores en huelga queda definitivamente prohibida, el alcance de esta disposición es limitado, toda vez que se introduce la idea de "servicios mínimos", para las funciones juzgadas vitales para las empresas durante los procesos de huelga, además de la posibilidad de establecer "pactos de adaptación" excepcionales. Por su parte, la disposición de titularidad sindical fue rechazada por el Tribunal Constitucional, frente a un nuevo requerimiento de parlamentarios de la derecha.

En este recorrido, la intervención del Tribunal Constitucional no es nueva. Ha sucedido de igual manera en otros ámbitos relevantes que, más allá del trabajo, tienen que ver con el derecho a la educación, el derecho al agua, el derecho de los consumidores, entre otros. No es de extrañar entonces que la revuelta popular que estalla en octubre de 2019, haya forzado al sistema político al establecimiento de un itinerario de cambio constitucional.

\section{PANORAMA CONTEMPORÁNEO DE LA TERCERIZACIÓN EN CHILE}

La pregunta que resta responder es en qué medida la ley 20.123 ha impactado en la realidad de la tercerización en Chile. Asumiendo que se trata de un fenómeno complejo, en lo que sigue se entregan algunos elementos generales que permiten reconstruir la situación contemporánea de la tercerización en el país.

De acuerdo a los datos de la Encuesta Nacional de Caracterización Laboral (ENCLA 2011, 2014) ${ }^{13}$, una primera constatación importante es que la tercerización sigue constituyendo una realidad del modelo productivo chileno, aun cuando se observan evoluciones recientes. Así, luego de haber alcanzado un nivel máximo en $2004(50,5 \%)$, la proporción de empresas que subcontratan disminuye progresivamente. Si bien en 2011, ella alcanza todavía una proporción importante (37,8\% de las empresas), ya en 2014 pasa a 17,6\%. Para el suministro temporal de trabajadores, seestima que en 2006 9,4\% de las empresas recurría a este mecanismo, marcando el nivel más elevado de esta práctica, para luego caer en los años siguientes: $2,8 \%$ en $2008,3,6 \%$ en 2011 y 1,2\% en 2014.

Con todo, pese a la disminución, es preciso notar que estas estimaciones hacen referencia a la proporción de empresas que recurren a la tercerización. Otra manera de aproximarse al fenómeno consiste en observar la cantidad de fuerza de trabajo tercerizada, lo que pareciera evidenciar una tendencia diferente. Las estimaciones de la Encuesta Nacional de Empleo del Instituto Nacional de Estadística (INE)

${ }^{13}$ La ENCLA 2019 debería ser publicada durante el segundo semestre del año 2020. 
entregan información más precisaa este respecto desde $2010^{14}$. En ese año, ésta alcanzaba 593.402 personas y representaba 11,5\% del total de la fuerza de trabajo. Contingente que no ha dejado de crecer tanto en términos brutos como relativos: para el último trimestre de 2019, la fuerza de trabajo tercerizada alcanza 1.114.485 personas, representando $17,2 \%$ del total de la fuerza de trabajo. Junto con ello, llama también la atención la evolución de las formas de tercerización. El personal suministrado ha sido la categoría que más ha crecido en la última década, pasando de 68.774 personas en 2010 a 444.778 en 2019, esto es un crecimiento de $647 \%$. Por su parte, el personal subcontratado pasa de 509.137 personas a 635.713 durante el mismo período, registrando un crecimiento del 125\%. Si bien éste último sigue siendo estadísticamente más importante que el personal suministrado, la diferencia de ritmo de crecimiento altera la composición de la fuerza de trabajo tercerizada: si en 2010, el 85\% del empleo tercerizado era explicado por la subcontratación, esta proporción cae a $57 \%$ en 2019; por su parte, el suministro crece del $12 \%$ al $40 \%$ en este período ${ }^{15}$.

Considerando estos elementos, la magnitud de la tercerización parece ambivalente: la tercerización disminuye como práctica de las empresas, mientras que la fuerza de trabajo tercerizada aumenta progresivamente. Una interpretación posible sería que, lejos de una disminución, se está frente a una reconfiguración del fenómeno: las empresas utilizan menos la tercerización, pero de manera más intensiva, de manera que una proporción más reducida de empresas intermediarias moviliza una cantidad creciente de trabajadores y trabajadoras.

Sin embargo, más allá de esta primera constatación, otras tendencias permanecen. Es el caso de la distribución de la subcontratación según el tamaño de las empresas. De acuerdo a la ENCLA 2014, la práctica de la subcontratación en las grandes empresas alcanza el 40,7\%, proporción que disminuye progresivamente conforme disminuye el tamaño de las empresas. Otra tendencia que permanece es la presencia de la subcontratación en empresas de todas las regiones del país y también de todos los sectores productivos ${ }^{16}$. De la misma manera y como se ha mencionado más arriba, la ley 20.123 no estableció ningún límite respecto de las actividades susceptibles de ser externalizadas. En este sentido, se constata que la

\footnotetext{
${ }^{14}$ Cada uno de los datos presentados provenientes de la Encuesta Nacional de Empleo del INE han sido obtenidos a través del banco de datos de esta institución (www.bancodatosene.ine.cl).

${ }^{15}$ A ello se suma la fuerza de trabajo "enganchada", cuya participación dentro de la fuerza de trabajo tercerizada no supera el 3\% en este período, aun cuando en términos efectivos se duplica, pasando de 15.491 personas en 2010 a 33.994 en 2019.

${ }^{16}$ De acuerdo a la Encuesta Nacional de Empleo de 2019, la presencia de personal tercerizado es mayor al personal de planta en el sector minero (54\%), el que corresponde principalmente a subcontrato (48\%). Otro sector donde la fuerza de trabajo tercerizada es también relevante es el sector de suministro de gas y electricidad (41\%), también bajo la forma de subcontrato (34\%). Por el contrario, si bien los sectores de servicios de enseñanza y de salud son también relevantes desde el punto de vista de la fuerza de trabajo tercerizada ( $34 \%$ y $35 \%$, respectivamente), en este caso el suministro temporal es más relevante, aportando respectivamente $25 \%$ y $24 \%$ de la fuerza de trabajo del sector. En el resto de los sectores, la situación es mucho más variable con un promedio de $17 \%$ de participación del personal tercerizado.
} 
tercerización de la actividad principal sigue constituyendo una práctica dominante de las empresas en Chile. La misma ENCLA 2014 revela quela subcontratación de la actividad principal aumenta de $24,1 \%$ en 2011 a 39,6\% en 2014. De la misma manera, del total de fuerza de trabajo tercerizada, este tipo de actividades involucra el $48,9 \%$ según la misma fuente.

Otros datos revelan también la permanencia de un tipo de vinculación precaria entre las empresas. La ENCLA 2011 mostraba que la subcontratación reposaba mayoritariamente $(53,8 \%)$ en un acuerdo verbal entre las partes, lo que traduce un amplio margen de informalidad de la relación; asimismo, la duración del vínculo sería limitada: en el $50,4 \%$ de los casos es menor a 5 años y en $41,9 \%$ de los casos es menor a 12 meses. A esta constatación, la ENCLA 2014 agrega que las empresas subcontratadas son mayoritariamente de menor tamaño: 48,8\% tiene menos de 49 trabajadores y 32\% tiene menos de 10 trabajadores, es decir, 8 de cada 10 empresas subcontratadas son pequeñas o microempresas. Además, una proporción importante de este grupo recibe diversos apoyos de la empresa mandante, ya sea aportes materiales $(49,6 \%)$, financieros $(36,7 \%)$, tecnológicos y de formalización profesional (36,7\%). Un último dato relevante a este respecto es que del total de empresas subcontratadas, sólo $27,7 \%$ realiza sus funciones exclusivamente al exterior de la empresa mandante, la mayoría (51,7\%) realiza sus funciones al interior de ésta última.

A la hora de caracterizar más precisamente la fuerza de trabajo tercerizada, es importante señalar que hombres y mujeres no participan de la misma manera en la tercerización. De acuerdo a la ENCLA 2014, los hombres participan mayoritariamente en la subcontratación $(83,1 \%)$, más en aquella que implica la actividad principal (95\%), mientras que las mujeres participan fundamentalmente en el suministro temporal de trabajo $(60,4 \%)$. Esta distribución se corrobora con los datos de la Encuesta Nacional de Empleo de 2019, ya que si bien la fuerza de trabajo femenina tercerizada se distribuye en proporciones menos extremas entre el subcontrato (41\%) y el suministro temporal (57\%), del total de la fuerza de trabajo subcontratada, la participación femenina alcanza $31 \%$, por el contrario, su presencia es mayoritaria en el suministro temporal (61\%). Esto último puede ser un indicador de mayor precariedad para la fuerza de trabajo femenina, en la medida que el suministro temporal constituye un régimen de trabajo más precario al ser estrictamente temporal y estar sometido a la triangulación laboral.

Otro indicador de mayor precariedad es la extensión de contratos de trabajo temporales o a plazo fijo. A este respecto, considerando el total de la fuerza de trabajo, sólo $29 \%$ tiene este tipo de contrato, proporción que disminuye en el caso del personal de planta (26\%). Lo contrario ocurre en la fuerza de trabajo tercerizada, ya que considerada en su conjunto, la proporción con este tipo de contrato alcanza $42 \%$, admitiendo leves variaciones en el conjunto del personal subcontratado $(40 \%)$ y suministrado (39\%). De esta manera, el empleo tercerizado tiene en la práctica el doble de probabilidades de tener un contrato a plazo fijo respecto del personal 
directamente contratado por la empresa en que trabaja. Finalmente, un último indicador de mayor precariedad dice relación con las brechas salariales entre personal de planta y tercerizado. De acuerdo a un estudio de Fundación Sol a partir de los datos de la Encuesta suplementaria de ingresos de 2017 del INE: "La brecha salarial (sin controles por variables observables) llega a los 109.974 pesos mensuales. En términos porcentuales, los trabajadores directamente contratados perciben en promedio un $21,4 \%$ más que los trabajadores externalizados. En la Región Metropolitana la brecha es de 60,2\%" (FUNDACIÓN SOL, 2018, p. 15). También se observan variaciones de la brecha salarial promedio entre personal de planta y tercerizado por sectores: mientras que en el comercio y la industria la brecha alcanza 30,6\% y 40,3\%, respectivamente, ésta aumenta en minería a 70,6\%, alcanzando valores extremos en las actividades financieras (163,6\%) y las actividades profesionales, científicas y técnicas (167\%).

A partir de estas estimaciones es posible definir un tipo ideal de las relaciones de subcontratación en Chile, marcadas mayoritariamente por la informalidad y la corta duración, además de un grado de verticalidad importante. En efecto, son mayoritariamente grandes empresas las que subcontratan sus actividades - de manera importante aquellas que forman parte del giro principal - a empresas de menor tamaño, cuyo nivel de autonomía material y financiera es más bien débil y realizan sus funciones al interior de las empresas mandantes. Asimismo, la fuerza de trabajo tercerizada tiene más probabilidades de ser precaria que el personal del planta, al menos en materia de inestabilidad de empleo y de retribuciones salariales. Desigualdades que se superponen a las brechas de género que también atraviesan el mercado del trabajo.

Pese a la regulación de la tercerización, la imagen global tiende a ser la misma que describían las investigaciones de los años 1990 y 2000. En ello influyen los elementos problemáticos que hemos destacado anteriormente, en la medida que no se asegura igualdad de condiciones, no se limitan las actividades objeto de externalización, se mantiene un concepto de empresa que refuerza la fragmentación del trabajo y la Dirección del Trabajo no cuenta con las atribuciones necesarias para su actividad fiscalizadora. Por estas razones, lejos de limitar la tercerización - al menos en cuanto a la magnitud de fuerza de trabajo implicada la ley 20.123 ha permitido su consolidación como régimen de trabajo precario.

\section{CONCLUSIÓN}

El objetivo central de este artículo ha sido reconstruir analíticamente el proceso de expansión y consolidación de la tercerización en Chile, buscando evidenciar el rol determinante de la legislación laboral en este proceso. A partir de este análisis es posible destacar que lejos de ser una práctica accesoria, la tercerización constituye una estrategia fundamental de la reconversión productiva operada en Chile luego de los ajustes estructurales neoliberales y de la adaptación contemporánea de las empresas, involucrando un volumen creciente de fuerza de 
trabajo. En segundo lugar, la regulación de la tercerización constituye un avance limitado respecto de las condiciones de trabajo, toda vez que deja vacíos importantes que redundan en una institucionalización de la desigualdad de condiciones entre el personal de planta y la fuerza de trabajo externa. En este sentido, esta regulación consolida el modelo dominante de tercerización, con relaciones verticales y dependientes entre las empresas y efectos de precarización para las y los trabajadores.

Por otra parte, un segundo objetivo de este análisis ha sido observar el fenómeno de la tercerización como operador analítico de las transformaciones más generales del trabajo en Chile. La observación de la (des)regulación de la tercerización a la luz de los cambios más amplios de la legislación laboral ha permitido evidenciar no sólo la afinidad entre ambos niveles de la regulación laboral, sino también lo que podríamos llamar una contextualización normativa de la regulación de la tercerización, a partir de la cual se especifican sus consecuencias concretas. En este sentido, la tercerización ocupa no sólo un lugar central en la estructura productiva, revela también las tensiones entre las orientaciones fundamentales de la legislación laboral. Los contextos de tercerización laboral constituyen el teatro de un encuentro crítico entre la masiva flexibilización unilateral de los contratos de trabajo y las limitaciones que aún pesan en materia sindical, reforzando la fragmentación de los colectivos laborales. No pareciera ser casualidad entonces que los últimos conflictos laborales de gran magnitud hayan emergido precisamente en estos contextos.

La coyuntura actual encuentra a Chile atravesado por un proceso constituyente abierto por la revuelta de octubre de 2019 y por los efectos devastadores de la pandemia de Covid-19. Es un período de cambios que tocan directamente los mundos del trabajo, cuyas consecuencias en el largo plazo son aún imprevisibles.

\section{REFERENCIAS BIBLIOGRAFICAS}

ABDELNOUR, S.; MEDA, D. Les nouveaux travailleurs des applis. Paris: Presses Universitaires de France, 2019.

ABRAMO, L.; MONTERO, C.; REINECKE, G., Cambios tecnológicos, encadenamientos productivos y calificaciones del trabajo en Chile: un balance. In: NOVICK, M. GALLART, M. (Eds.). Competitividad, redes productivas y competencias laborales: ¿homogeneidad o segmentación? Montevideo, OIT, 1997.

AGACINO, R., Pasado y presente: los trabajadores una vez más. Disponible en: www.redem.buap.mx

AGACINO, R., GONZÁLEZ, C., ROJAS, J. Capital transnacional y trabajo: el desarrollo minero en Chile.Santiago. LOM-PET, 1998. 
AGACINO, R.; RIVAS, G., La industria chilena después del ajuste: evaluación y perspectivas. In: OIT. La industria chilena: ajuste, evolución, innovaciones y perspectivas. Santiago, 1995.

ARAVENA, A.; NÚÑEZ, D. El renacer de la huelga obrera en Chile. Santiago, ICAL, 2009.

ARTEAGA, I. En busca del concepto jurídico de empresa. Revista Chilena de Derecho, v. 29, n. 3, 2002, p. 603-620.

BEROUD, S.; BOUFFARTIGUE, P. Quand le travail se précarise, quelles résistances collectives? Paris: La Dispute, 2009.

BASUALDO, V.; MORALES, D. La tercerización laboral: orígenes, impacto y claves para su análisis en América Latina. Buenos Aires: Siglo XXI, 2014.

BATTISTINI, O. ¿Qué hay de nuevo y de viejo en la subcontratación laboral moderna? Sociológica, a. 33, n. 93, enero-abril de 2018, p. 281-318.

BIBLIOTECA DEL CONGRESO NACIONAL. Historia de la Ley 20.123. Santiago.

Disponible en: www.bcn.cl

BOURDIEU, P. Les structures sociales de l'économie. Paris: Seuil, 2000.

CAMPERO, G. La economía política de las relaciones laborales: 1990-2006. CIEPLAN, Santiago, 2007.

CAMPERO, G.; VALENZUELA, J. El movimiento sindical en el régimen militar chileno: 1973-1981. Instituto Latinoamericano de Estudios del Trabajo ILET, Santiago, 1984.

CORIAT, B., L'atelier et le robot. Essai sur le fordisme et la production de masse à l'âge de l'électronique. Paris: Christian Bourgois Éditeur, 1990.

DÍAZ, A. La industria chilena entre 1970-1994: de la sustitución de importaciones a la segunda fase exportadora. Santiago: CEPAL, 1995.

DIDRY, C. L'institution du travail. Droit et salariat dans l'histoire. Paris: La Dispute, 2016. 
DRAKE, P. El movimiento obrero en Chile: De la Unidad Popular a la Concertación. Revista de Ciencia Política, n. 2, v. 23, Santiago, 2003, p. 148-158.

ECHEVERRÍA, M. La historia inconclusa de la subcontratación, Dirección del Trabajo. Santiago, 2010.

ECHEVERRÍA, M. Los riesgos laborales de la subcontratación, Dirección del Trabajo. Santiago, 1997.

ENCLA. Informe de resultados octava encuesta laboral. Dirección del Trabajo, Santiago, 2014.

ENCLA. Informe de resultados séptima encuesta laboral. Dirección del Trabajo, Santiago, 2011.

ENCLA. Informe de resultados quinta encuesta laboral. Dirección del Trabajo, Santiago, 2006.

ESCOBAR, P., LÓPEZ, D. El sector forestal en Chile: crecimiento y precarización del empleo.Santiago. PET, 1996.

FFRENCH-DAVIS, R.; STALLINGS, B. Reformas, crecimiento y políticas sociales en Chile desde 1973. Santiago: LOM-CEPAL, 2001.

FOUCAUTL, M. La naissance de la biopolitique. Paris: Seuil, 2004.

FUNDACIÓN SOL. Los verdaderos sueldos de Chile.Santiago, 2017. Disponible en: www.fundacionsol.cl

GÁRATE, M. La « révolution économique » au Chili. A la recherche de l'utopie néoconservatrice 1973-2003. Thèse de doctorat en Histoire : École des hautes études en sciences sociales, Paris, 2010.

GARRETÓN, M.; CRUZ, M.; AGUIRRE, F. La experiencia de los consejos asesores presidenciales en Chile y la construcción de los problemas públicos. Revista Mexicana de Sociología, v. 74, n. 2, abril-junio, 2012, p. 303-340. 
GARRETÓN, M. Neoliberalismo corregido y progresismo limitado. Los gobiernos de la Concertación en Chile, 1990-2010. Santiago: ARCIS-CLACSOPROSPAL, 2012.

GARRETÓN, M. De la transición a los problemas de calidad de la democracia chilena. Revista Política, Santiago, v. 2, otoño, 2004.

INSTITUTO CHILENO DEL ACERO. Análisis del proceso de subcontratación en el sector de la Línea blanca en Chile. Documento de Trabajo no 159, Santiago: OITPREALC, 1978.

LÓPEZ, D. Subcontratación y conflictos laborales. Santiago: Universidad Central, 2008.

MARTÍNEZ, J.; DÍAZ, Á. Chile: The great transformation. Washington: The Brookings Institution, 1996.

MARX, K. Le Capital. Paris: Presses Universitaires de France, 1993.

MONTERO, C. Les nouveaux entrepreneurs: le cas du Chili. Paris: L'Harmattan, 1997.

MONTERO, C. Travail et travailleurs au Chili. Paris: La Découverte, 1983.

NARBONA, K. (2015), Antecedentes del modelo de relaciones laborales chileno. Fundación SOL. Disponible en: www.fundacionsol.cl

OBSERVATORIO DE HUELGAS LABORALES. Informe de huelgas laborales 2016. Santiago, Chile: COES-UAH, 2017. Disponible en: http://www.coes.cl/

PÉREZ SEPÚLVEDA, S. À l'ombre de la sous-traitance. Reconfigurations politiques du travail et des travailleurs au Chili. Thèse de doctorat en sociologie. Paris: École des hautes études en sciences sociales, 2018.

PIÑERA, J. La revolución laboral en Chile. Santiago: Zig-Zag, 1900.

RAMOS, C. La transformación de la empresa chilena. Una modernización desbalanceada. Santiago: Universidad Alberto Hurtado, 2009. 
ROJAS,I., Las reformas laborales al modelo normativo de negociación colectiva del Plan Laboral. Ius et Praxis, v. 13, n.2, 2007, p. 195-221.

UGARTE, J. El trabajador en su soledad: el modelo de relaciones laborales chileno y la promesa incumplida. In: A. STECHER, A.; GODOY, L. (dir.). Transformaciones del trabajo, subjetividad e identidades: lecturas psicosociales desde Chile y América Latina. Santiago: RIL Editores, 2014.

UGARTE, J. El concepto legal de empresa y el derecho laboral: cómo salir del laberinto. Revista Chilena de Derecho Privado, n. 20, 2013, p. 185-213.

WEIL, D. The fissured workplace. Why work became so bad for so many and what can be done to improve it. Harvard University Press, 2017.

WORMALD, G. De las pirámides a las redes. In: ALBUQUERQUE, M., MONTERO, C., ENSIGNIA, J. (Eds). Trabajo y empresa: entre dos siglos. Caracas, Nueva Sociedad, 1999. 